\title{
Complete Controllability of Fractional Neutral Differential Systems in Abstract Space
}

\author{
Fang Wang, ${ }^{1}$ Zhen-hai Liu, ${ }^{2}$ and Jing $\mathrm{Li}^{3}$ \\ ${ }^{1}$ School of Mathematics and Computing Science, Changsha University of Science and Technology, \\ Changsha, Hunan Province 410076, China \\ ${ }^{2}$ School of Mathematics and Computer Science, Guangxi University for Nationalities, Nanning, Guangxi Province 530006, China \\ ${ }^{3}$ Changsha University of Science and Technology, Changsha, Hunan, China
}

Correspondence should be addressed to Fang Wang; wangfang811209@tom.com

Received 10 September 2012; Revised 9 November 2012; Accepted 10 November 2012

Academic Editor: Yong Zhou

Copyright (C) 2013 Fang Wang et al. This is an open access article distributed under the Creative Commons Attribution License, which permits unrestricted use, distribution, and reproduction in any medium, provided the original work is properly cited.

By using fractional power of operators and Sadovskii fixed point theorem, we study the complete controllability of fractional neutral differential systems in abstract space without involving the compactness of characteristic solution operators introduced by us.

\section{Introduction}

Recently, fractional differential systems have been proved to be valuable tools in the modeling of many phenomena in various fields of science and engineering. Indeed, we can find numerous applications in viscoelasticity, electrochemistry, control, porous media, electromagnetic, and so forth (see [15]). There has been a great deal of interest in the solutions of fractional differential systems in analytic and numerical sense. One can see the monographs of Kilbas et al. [6], Miller and Ross [7], Podlubny [8], Lakshmikantham et al. [9], Tarasov [10], Wang et al. [11-13] and the survey of Agarwal et al. [14] and the reference therein. In order to study the fractional systems in the infinite dimensional space, the first important step is how to introduce a new concept of mild solutions. A pioneering work has been reported by EI-Borai [15] and Zhou and Jiao [16].

In recent years, controllability problems for various types of nonlinear fractional dynamical systems in infinite dimensional spaces have been considered in many publications. An extensive list of these publications focused on the complete and approximate controllability of the fractional dynamical systems can be found (see [17-34]). Although the controllability of fractional differential systems in abstract space has been discussed, Hernández et al. [35] point out that some papers on controllability of abstract control systems contain a similar technical error when the compactness of semigroup and other hypotheses is satisfied, more precisely, in this case the application of controllability results are restricted to the finite dimensional space. Ji et al. [32] find some conditions guaranteeing the controllability of impulsive differential system when the Banach space is nonseparable and evolution systems are not compact, by means of Möch fixed point theorem and the measure of noncompactness. Meanwhile, Wang et al. $[19,20]$ have researched the complete controllability of fractional evolution systems without involving the compactness of characteristic solution operators. Neutral differential equations arise in many areas of applied mathematics and for this reason these equations have received much attention in the last decades. Sakthivel and Ren [29] have established a new set of sufficient conditions for the complete controllability for a class of fractional order neutral systems with bounded delay under the natural assumption that the associated linear control is completely controllable. To the author's knowledge, there are few papers on the complete controllability of the abstract neutral fractional differential systems with unbounded delay.

In the present paper, we introduce a suitable concept of the mild solutions including characteristic solution operators $\varphi(\cdot)$ and $S(\cdot)$ which are associated with operators semigroup $\{T(t) ; t \geq 0\}$ and some probability density functions $\xi_{q}$. Then also without involving the compactness of characteristic solution operators, we obtain the controllability of the 
following abstract neutral fractional differential systems with unbounded delay:

$$
\begin{gathered}
{ }^{c} D_{t}^{q}\left(x(t)+F\left(t, x_{t}\right)\right)+A x(t)=C u(t)+G\left(t, x_{t}\right), \\
t \in(0, a], \\
x_{0}(\vartheta)=\phi(\vartheta) \in B, \quad \vartheta \in(-\infty, 0],
\end{gathered}
$$

where the state variable $x(\cdot)$ takes values in Banach space $X, x_{t}:(-\infty, 0] \rightarrow X, x_{t}(\vartheta)=x(t+\vartheta)$ belongs to some abstract phase space $B$, and $B$ is the phase space to be specified later. The control function $u(\cdot)$ is given in $L^{2}([0, a] ; U)$, with $U$ as a Banach spaces. $C$ is a bounded linear operator from $U$ to $X$. The operator $-A$ is a generator of a uniformly bounded analytic semigroup $\{T(t), t \geq 0\}$ in which $X, F, G:[0, a] \times$ $B \rightarrow X$ are appropriate functions.

\section{Preliminaries}

Throughout this paper $X$ will be a Banach space with norm $\|\cdot\|$ and $Y$ is another Banach space, $L_{b}(X, Y)$ denote the space of bounded linear operators from $X$ to $Y$. We also use $\|f\|_{L^{p}\left([0, a], R^{+}\right)}$to denote the $L^{p}\left([0, a], R^{+}\right)$of norm of $f$ whenever $f \in L^{p}\left([0, a], R^{+}\right)$for some $p$ with $1 \leq p<\infty$. Let $L^{p}\left([0, a], R^{+}\right)$denote the Banach space of functions $f:[0, a] \rightarrow X$ which are Bochner integrable normed by $\|f\|_{L^{p}\left([0, a], R^{+}\right)}$. Let $-A: D(A) \rightarrow X$ be the infinitesimal generator of a uniformly bounded analytic semigroup $T(t)$. Let $0 \in \rho(A)$, then it is possible to define the fractional power $A^{\alpha}$, for $0<\alpha \leq 1$, as a closed linear operator on its domain $D\left(A^{\alpha}\right)$. Furthermore, the subspace $D\left(A^{\alpha}\right)$ is dense in $X$ and the expression

$$
\|x\|_{\alpha}=\left\|A^{\alpha} x\right\|, \quad x \in D\left(A^{\alpha}\right)
$$

defines a norm on $D\left(A^{\alpha}\right)$. Hereafter we denote by $X_{\alpha}$ the Banach space $D\left(A^{\alpha}\right)$ normed with $\|x\|_{\alpha}$. Then for each $0<$ $\alpha \leq 1, X_{\alpha}$ the Banach space, and $\|x\|_{\alpha} \hookrightarrow\|x\|_{\beta}$ for $0<\beta<$ $\alpha \leq 1$ and the imbedding is compact whenever the resolvent operator of $A$ is compact. For a uniformly bounded analytic semigroup $\{T(t) ; t \geq 0\}$ the following properties will be used:

(a) there is a $M \geq 0$ such that $\|T(t)\| \leq M$ for all $t \geq 0$.

(b) for any $\alpha \geq 0$, there exists a positive constant $C_{\alpha}$ such that

$$
\left\|A^{\alpha} T(t)\right\| \leq \frac{C_{\alpha}}{t^{\alpha}}, \quad 0<t \leq a
$$

For more details about the above preliminaries, we can refer to [16].

Although the semigroup $\{T(t) ; t \geq 0\}$ is only the uniformly bounded analytic semigroup but not compact, we can also give the definition of mild solution for our problem by using the similar method introduced in [36].

Definition 1. We say that a function $x(\cdot):(-\infty, a] \rightarrow X$ is a mild solution of the system (1) if $x_{0}=\phi$, the restriction of $x(\cdot)$ to the interval $[0, a]$ is continuous and for each $0 \leq t \leq a$, the function $A S(t-s) F\left(s, x_{s}\right), s \in[0, t]$ is integrable and satisfies the following integral equation:

$$
\begin{aligned}
x(t)= & \varphi(t)[\phi(0)+F(0, \phi)]-F\left(t, x_{t}\right) \\
& -\int_{0}^{t}(t-s)^{q-1} A S(t-s) F\left(s, x_{s}\right) d s \\
& +\int_{0}^{t}(t-s)^{q-1} S(t-s)\left[C u(s)+G\left(s, x_{s}\right)\right] d s,
\end{aligned}
$$

where $\varphi(t)$ and $S(t)$ are called characteristic solution operators and are given by

$$
\begin{aligned}
& \varphi(t)=\int_{0}^{\infty} \xi_{q}(\theta) T\left(t^{q} \theta\right) d \theta, \\
& S(t)=q \int_{0}^{\infty} \theta \xi_{q}(\theta) T\left(t^{q} \theta\right) d \theta,
\end{aligned}
$$

and for $\theta \in(0, \infty), \xi_{q}(\theta)=(1 / q) \theta^{-1-1 / q} \bar{w}_{q}\left(\theta^{-1 / q}\right) \geq 0$,

$$
\bar{w}_{q}(\theta)=\frac{1}{\pi} \sum_{n=1}^{\infty}(-1)^{n-1} \vartheta^{-q n-1} \frac{\Gamma(n q+1)}{n !} \sin (n \pi q) .
$$

Here, $\xi_{q}$ is a probability density function defined on $(0, \infty)$, that is, $\xi_{q}(\theta) \geq 0, \theta \in(0, \infty)$, and $\int_{0}^{\infty} \xi_{q}(\theta) d \theta=1$.

Definition 2 (complete controllability). The fractional system (1) is said to be completely controllable on the interval $[0, a]$ if, for every initial function $\phi \in B$ and $x_{1} \in X$ there exists a control $u \in L^{2}([0, a], U)$ such that the mild solution $x(\cdot)$ of (1) satisfies $x(a)=x_{1}$.

The following results of $\varphi(t)$ and $S(t)$ will be used throughout this paper.

Lemma 3. The operators $\varphi(t)$ and $S(t)$ have the following properties:

(i) for any fixed $t \geq 0, \varphi(t)$ and $S(t)$ are linear and bounded operators, that is, for any $x \in X$,

$$
\begin{gathered}
\|\varphi(t) x\| \leq M_{0}\|x\|, \\
\|S(t) x\| \leq \frac{q M_{0}}{\Gamma(1+q)}\|x\| ;
\end{gathered}
$$

(ii) $\{\varphi(t), t \geq 0\}$ and $\{S(t), t \geq 0\}$ are strongly continuous and there exists $M_{1}, M_{2}$ such that $\|\varphi(t)\| \leq M_{1}$, $\|S(t)\| \leq M_{2}$ for any $t \in[0, a]$

(iii) for $t \in[0, a]$ and any bounded subsets $D \subset X, t \rightarrow$ $\{\varphi(t) x: x \in D\}$ and $t \rightarrow\{S(t) x: x \in D\}$ are equicontinuous if $\left\|T\left(t_{2}^{q} \theta\right) x-T\left(t_{1}^{q} \theta\right) x\right\| \rightarrow 0$ with respect to $x \in D$ as $t_{2} \rightarrow t_{1}$ for each fixed $\theta \in[0, \infty]$.

The proof of Lemma 3 we can see in [33].

To end this section, we recall Kuratowski's measure of noncompactness, which will be used in the next section to study the complete controllability via the fixed points of condensing operator. 
Definition 4. Let $X$ be a Banach space and $\Omega_{X}$ the bounded set of $X$. The Kuratowski's measure of noncompactness is the map $\alpha: \Omega_{X} \rightarrow[0, \infty)$ defined by

$$
\alpha(D)=\inf \left\{d>0: D \subseteq \bigcup_{i=1}^{n} D_{i}, \operatorname{diam}\left(D_{i}\right) \leq d\right\}
$$

here $D \in \Omega_{X}$.

One will use the following basic properties of the $\alpha$ measure and Sadovskii's fixed point theorem here (see [3739]).

Lemma 5. Let $D_{1}$ and $D_{2}$ be two bounded sets of a Banach space $X$. Then

(i) $\alpha\left(D_{1}\right)=0$ if and only if $D_{1}$ is relatively compact;

(ii) $\alpha\left(D_{1}\right) \leq \alpha\left(D_{2}\right)$ if $D_{1} \subseteq D_{2}$;

(iii) $\alpha\left(D_{1}+D_{2}\right) \leq \alpha\left(D_{1}\right)+\alpha\left(D_{2}\right)$.

Lemma 6 (sadovskii's fixed point theorem). Let $N$ be a condensing operator on a Banach space $X$, that is, $N$ is continuous and takes bounded sets into bounded sets, and $\alpha(N(D))<\alpha(D)$ for every bounded set $D$ of $X$ with $\alpha(D)>0$. If $N(S) \subset S$ for a convex closed and bounded set $S$ of $X$, then $N$ has a fixed point in $S$.

\section{Complete Controllability Result}

To study the system (1), we assume the function $x_{t}$ represents the history of the state from $-\infty$ up to the present time $t$ and $x_{t}:(-\infty, 0] \rightarrow X, x_{t}(\vartheta)=x(t+\vartheta)$ belongs to some abstract phase space $B$, which is defined axiomatically. In this article, we will employ an axiomatic definition of the phase space $B$ introduced by Hale and Kato [40] and follow the terminology used in [41]. Thus, $B$ will be a linear space of functions mapping $(-\infty, 0]$ into $X$ endowed with a seminorm $\|\cdot\|_{B}$. We will assume that $B$ satisfies the following axioms:

(A) If $x \in(-\infty, \sigma+a) \rightarrow X, a>0$, is continuous on $[\sigma, \sigma+a]$ and $x_{\sigma} \in B$, then for every $t \in[\sigma, \sigma+a]$ the following conditions hold:

(i) $x_{t}$ is in $B$;

(ii) $\|x(t)\| \leq H\left\|x_{t}\right\|_{B}$;

(iii) $\left\|x_{t}\right\|_{B} \leq K(t-\sigma) \sup \{\|x(t)\|: \sigma \leq s \leq t\}+M(t-$ $\sigma)\left\|x_{\sigma}\right\|_{B}$.

Here $H \geq 0$ is a constant, $K, M:[0,+\infty) \rightarrow[0,+\infty)$, $K$ is continuous and $M$ is locally bounded, and $H, K, M$ are independent of $x(t)$.

(B) For the function $x(\cdot)$ in $(\mathrm{A}), x_{t}$ is a $B$-valued continuous function on $[\sigma, \sigma+a]$.

(C) The space $B$ is complete.

Now we give the basic assumptions on the system (1).

$\left(H_{0}\right)$ (i) A generates a uniformly bounded analytic semigroup $\{T(t), t \geq 0\}$ in $X$; (ii) for all bounded subsets
$D \subset X$ and $x \in D,\left\|T\left(t_{2}^{q} \theta\right) x-T\left(t_{1}^{q} \theta\right) x\right\| \rightarrow 0$ as $t_{2} \rightarrow t_{1}$ for each fixed $\theta \in[0, \infty]$.

$\left(H_{1}\right) F:[0, a] \times B \rightarrow X$ is continuous function, and there exists a constant $\beta \in(0,1)$ and $L, L_{1}>0$ such that the function $F$ is $X_{\beta}$-valued and satisfies the Lipschitz condition:

$$
\begin{aligned}
& \left\|A^{\beta} F\left(s_{1}, \phi_{1}\right)-A^{\beta} F\left(s_{2}, \phi_{2}\right)\right\| \\
& \quad \leq L\left(\left|s_{1}-s_{2}\right|+\left\|\phi_{1}-\phi_{2}\right\|_{B}\right),
\end{aligned}
$$

for $0 \leq s_{1}, s_{2} \leq a, \phi_{1}, \phi_{2} \in B$, and the inequality

$$
\left\|A^{\beta} F(t, \phi)\right\| \leq L_{1}\left(\|\phi\|_{B}+1\right)
$$

holds for $t \in[0, a], \phi \in B$.

$\left(H_{2}\right)$ The function $G:[0, a] \times B \rightarrow X$ satisfies the following conditions:

(i) for each $t \in[0, a]$, the function $G(t, \cdot): B \rightarrow X$ is continuous and for each $\phi \in B$ the function $G(\cdot, \phi)$ : $[0, a] \rightarrow X$ is strongly measureable;

(ii) for each positive number $k$, there is a positive function $g_{k} \in L^{1 / q_{1}}([0, a]), 0<q_{1}<q$ such that

$$
\begin{gathered}
\sup _{\|\phi\|_{B} \leq k}\|G(t, \phi)\| \leq g_{k}(t), \\
\liminf \frac{1}{k}\left\|g_{k}\right\|_{L^{1 / q_{1}[0, a]}}=\gamma<\infty .
\end{gathered}
$$

$\left(\mathrm{H}_{3}\right)$ The linear operator $C$ is bounded, $W$ from $U$ into $X$ is defined by

$$
W u=\int_{0}^{a}(a-s)^{q-1} S(a-s) C u(s) d s
$$

and there exists a bounded invertible operator $W^{-1}$ defined on $L^{2}([0, a] ; U) / \operatorname{ker} W$ and there exist two positive constants $M_{3}, M_{4}>0$ such that $\|B\|_{L_{b}(U, X)} \leq$ $M_{3},\left\|W^{-1}\right\|_{L_{b}\left(X, L^{2}([0, a], U) / \operatorname{ker} W\right)} \leq M_{4}$.

$\left(H_{4}\right)$ For all bounded subsets $D \subseteq X$, the set

$$
\Pi_{h, \delta}(t)=\left\{Q_{2, h, \delta} z(t) \mid z \in D\right\}
$$

where

$$
\begin{array}{r}
Q_{2, h, \delta} z(t)=\int_{0}^{t-h} \int_{\delta}^{\infty}(t-s)^{q-1} \theta \xi_{q}(\theta) q T\left((t-s)^{q} \theta\right) \\
\times\left[C u(s)+G\left(s, \bar{z}_{s}+y_{s}\right) d \theta d s\right.
\end{array}
$$

is relatively compact in $X$ for arbitrary $h \in(0, t)$ and $\delta>0$. 
Theorem 7. Let $\phi \in B$. If the assumptions $\left(H_{0}\right)-\left(H_{4}\right)$ are satisfied, then the system (1) is controllable on interval $[0, a]$ provided that

$$
\begin{gathered}
M_{5} L K_{a}+\frac{C_{1-\beta} \Gamma(1+\beta) a^{q \beta}}{\beta \Gamma(1+q \beta)} L K_{a}<1, \\
\left(1+a M_{2} M_{3} M_{4}\right) \\
\times\left(L_{1} M_{5} K_{a}+M_{2}\left(\left(\frac{1-q_{1}}{q-q_{1}}\right) a^{\left(q-q_{1}\right) /\left(1-q_{1}\right)}\right)^{1-q_{1}} K_{a} \gamma\right. \\
\left.+\frac{C_{1-\beta} \Gamma(1+\beta) a^{q \beta}}{\beta \Gamma(1+q \beta)} L_{1} K_{a}\right)<1,
\end{gathered}
$$

where $M_{5}=\left\|A^{-\beta}\right\|, K_{a}=\sup \{K(t): 0 \leq t \leq a\}$ and $C_{1-\beta}$ is from (3).

Proof. Using the assumption $\left(H_{3}\right)$, for arbitrary function $x(\cdot)$ define the control

$$
\begin{aligned}
u(t)=W^{-1}\left[x_{1}\right. & -\varphi(a)(\phi(0)+F(0, \phi))+F\left(a, x_{a}\right) \\
& +\int_{0}^{a}(a-s)^{q-1} A S(a-s) F\left(s, x_{s}\right) d s \\
& \left.-\int_{0}^{a}(a-s)^{q-1} S(a-s) G\left(s, x_{s}\right) d s\right](t) .
\end{aligned}
$$

It will be shown that when using this control the operator $P$ defined by

$$
\begin{aligned}
P x(t)= & \varphi(t)[\phi(0)+F(0, \phi)]-F\left(t, x_{t}\right) \\
& -\int_{0}^{t}(t-s)^{q-1} A S(t-s) F\left(s, x_{s}\right) d s \\
& +\int_{0}^{t}(t-s)^{q-1} S(t-s)\left[C u(s)+G\left(s, x_{s}\right)\right] d s
\end{aligned}
$$

has a fixed point $x(\cdot)$. Then $x(\cdot)$ is a mild solution of system (1), and it is easy to verify that $x(a)=P x(a)=x_{1}$, which implies that the system is controllable.

Next we will prove that $P$ has a fixed point using the fixed point theorem of Sadovskii [38].

Let $y(\cdot):(-\infty, a] \rightarrow X$ be the function defined by

$$
y(t)= \begin{cases}\varphi(t) \phi(0), & t \in[0, a], \\ \phi(t), & -\infty<t<0,\end{cases}
$$

then $y_{0}=\phi$ and the map $t \rightarrow y_{t}$ is continuous. We can assume $N=\sup \left\{\left\|y_{t}\right\|: 0 \leq t \leq a\right\}$. For each $z \in C([0, a]$ : $X), z(0)=0$. We can denote by $\bar{z}$ the function defined by

$$
\bar{z}(t)= \begin{cases}z(t), & 0 \leq t \leq a, \\ 0, & -\infty<t<0 .\end{cases}
$$

If $x(\cdot)$ satisfies (18), we can decompose it as $x(t)=\bar{z}(t)+y(t)$, $0 \leq t \leq a$, which implies $x_{t}=\bar{z}_{t}+y_{t}$ for every $0 \leq t \leq a$ and the function $z(\cdot)$ satisfies

$$
\begin{aligned}
z(t)= & \varphi(t) F(0, \phi)-F\left(t, \bar{z}_{t}+y_{t}\right) \\
& -\int_{0}^{t}(t-s)^{q-1} A S(t-s) F\left(s, \bar{z}_{s}+y_{s}\right) d s \\
& +\int_{0}^{t}(t-s)^{q-1} S(t-s)\left[C u(s)+G\left(s, \bar{z}_{s}+y_{s}\right)\right] d s
\end{aligned}
$$

Moreover $\bar{z}_{0}=0$. Let $Q$ be the operator on $C([0, a], X)$ defined by

$$
\begin{aligned}
Q z(t)= & \varphi(t) F(0, \phi)-F\left(t, \bar{z}_{t}+y_{t}\right) \\
& -\int_{0}^{t}(t-s)^{q-1} A S(t-s) F\left(s, \bar{z}_{s}+y_{s}\right) d s \\
& +\int_{0}^{t}(t-s)^{q-1} S(t-s)\left[C u(s)+G\left(s, \bar{z}_{s}+y_{s}\right)\right] d s .
\end{aligned}
$$

Obviously the operator $P$ has a fixed point is equivalent to $Q$ has a fixed point, so it turns out to prove that $Q$ has a fixed point. For each positive number $k$, let

$$
B_{k}=\{z \in C([0, a]: X): z(0)=0,\|z(t)\| \leq k, 0 \leq t \leq a\},
$$

then for each $k, B_{k}$ is clearly a bounded closed convex set in $C([0, a]: X)$. Since by (3) and (10) the following relation holds:

$$
\begin{aligned}
\left\|A S(t-s) F\left(s, \bar{z}_{s}+y_{s}\right)\right\| \leq & \left\|A^{1-\beta} S(t-s) A^{\beta} F\left(s, \bar{z}_{s}+y_{s}\right)\right\| \\
\leq & \frac{C_{1-\beta} q \Gamma(1+\beta)}{\Gamma(1+q \beta)}(t-s)^{-(1-\beta) q} \\
& \times L_{1}\left(\left\|\bar{z}_{s}+y_{s}\right\|_{B}+1\right) \\
\leq & \frac{C_{1-\beta} q \Gamma(1+\beta)}{\Gamma(1+q \beta)}(t-s)^{-(1-\beta) q} \\
& \times L_{1}\left(\left\|\bar{z}_{s}\right\|_{B}+\left\|y_{s}\right\|_{B}+1\right) \\
\leq & \frac{C_{1-\beta} q \Gamma(1+\beta)}{\Gamma(1+q \beta)}(t-s)^{-(1-\beta) q} \\
& \times L_{1}\left(k K_{a}+N+1\right),
\end{aligned}
$$

then from Bocher's theorem [42] it follows that AS( $t$ s) $F\left(s, \bar{z}_{s}+y_{s}\right)$ is integrable on $[0, a]$, so $Q$ is well defined on $B_{k}$.

In order to make the following process clear we divide it into several steps.

Step 1 . We claim that there exists a positive number $k$ such that $Q\left(B_{k}\right) \subseteq B_{k}$. 
If it is not true, then for each positive number $k$, there is a function $z_{k}(\cdot) \in B_{k}$, but $Q z_{k} \notin B_{k}$, that is, $\left\|\mathrm{Q} z_{k}(t)\right\|>k$ for some $t \in[0, a]$. However, on the other hand, we have

$$
\begin{aligned}
& k<\left\|Q z_{k}(t)\right\| \\
& =\| \varphi(t) F(0, \phi)-F\left(t, \bar{z}_{k, t}+y_{t}\right) \\
& -\int_{0}^{t}(t-s)^{q-1} \\
& \times A S(t-s) F\left(s, \bar{z}_{k, s}+y_{s}\right) d s \\
& +\int_{0}^{t}(t-s)^{q-1} S(t-s) \\
& \times\left[C u_{k}(s)+G\left(s, \bar{z}_{k, s}+y_{s}\right)\right] d s \| \\
& =\| \varphi(t) F(0, \phi)-F\left(t, \bar{z}_{k, t}+y_{t}\right) \\
& -\int_{0}^{t}(t-s)^{q-1} A S(t-s) \\
& \times F\left(s, \bar{z}_{k, s}+y_{s}\right) d s \\
& +\int_{0}^{t}(t-s)^{q-1} S(t-s) \\
& \times\left[C W ^ { - 1 } \left\{x_{1}-\varphi(a)[\phi(0)+F(0, \phi)]\right.\right. \\
& +F\left(a, \bar{z}_{k, a}+y_{a}\right) \\
& +\int_{0}^{a}(a-\tau)^{q-1} A S(a-\tau) \\
& \times F\left(\tau, \bar{z}_{k, \tau}+y_{\tau}\right) d \tau \\
& -\int_{0}^{a}(a-\tau)^{q-1} S(a-\tau) \\
& \left.\times G\left(\tau, \bar{z}_{k, \tau}+y_{\tau}\right) d \tau\right\}(s) d s \\
& +\int_{0}^{t}(t-s)^{q-1} S(t-s) \\
& \left.\times G\left(s, \bar{z}_{k, s}+y_{s}\right)\right] d s \| \\
& \leq M_{1}\|F(0, \phi)\|+\left\|F\left(t, \bar{z}_{k, t}+y_{t}\right)\right\| \\
& +\int_{0}^{t}(t-s)^{q-1}\left\|A S(t-s) F\left(s, \bar{z}_{k, s}+y_{s}\right)\right\| d s \\
& +\int_{0}^{t} M_{2} M_{3} M_{4} \\
& \times\left\{\left\|x_{1}\right\|+M_{1} \| \phi(0) \text { is }+F(0, \phi) \|\right.
\end{aligned}
$$

$$
\begin{gathered}
+\left\|F\left(a, \bar{z}_{k, a}+y_{a}\right)\right\| \\
+\int_{0}^{a}(a-\tau)^{q-1} \\
\quad \times\left\|A S(a-\tau) F\left(\tau, \bar{z}_{k, \tau}+y_{\tau}\right)\right\| d \tau \\
+\int_{0}^{a} M_{2}(a-\tau)^{q-1} \\
\left.\times\left\|G\left(\tau, \bar{z}_{k, \tau}+y_{\tau}\right)\right\| d \tau\right\}(s) d s \\
+\int_{0}^{t} M_{2}(t-s)^{q-1}\left\|G\left(s, \bar{z}_{k, s}+y_{s}\right)\right\| d s,
\end{gathered}
$$

where $u_{k}$ is the corresponding control of $x_{k}, x_{k}=\bar{z}_{k}+y$. Since

$$
\begin{aligned}
\left\|\int_{0}^{t}(t-s)^{q-1} A S(t-s) F\left(s, \bar{z}_{k, s}+y_{s}\right) d s\right\| \\
\leq \int_{0}^{t}(t-s)^{q-1}\left\|A^{1-\beta} S(t-s) A^{\beta} F\left(s, \bar{z}_{k, s}+y_{s}\right)\right\| d s \\
\leq \frac{C_{1-\beta} q \Gamma(1+\beta)}{\Gamma(1+q \beta)} \\
\quad \times \int_{0}^{t}(t-s)^{q-1}(t-s)^{-(1-\beta) q} L_{1}\left(k K_{a}+N+1\right) \\
\leq \frac{C_{1-\beta} \Gamma(1+\beta) a^{q \beta}}{\beta \Gamma(1+q \beta)} L_{1}\left(k K_{a}+N+1\right), \\
\left\|F\left(t, \bar{z}_{k, t}+y_{t}\right)\right\| \\
=\left\|A^{-\beta} A^{\beta} F\left(t, \bar{z}_{k, t}+y_{t}\right)\right\| \\
\leq M_{5} L_{1}\left(k K_{a}+N+1\right), \\
\int_{0}^{t}\left\|(t-s)^{q-1} G\left(s, \bar{z}_{k, s}+y_{s}\right)\right\| d s \\
\leq \int_{0}^{t}(t-s)^{q-1} g_{k K_{a}+N}(s) d s,
\end{aligned}
$$

there holds

$$
\begin{aligned}
& k<M_{1}\|F(0, \phi)\|+M_{5} L_{1}\left(k K_{a}+N+1\right) \\
& +\frac{C_{1-\beta} \Gamma(1+\beta) a^{q \beta}}{\beta \Gamma(1+q \beta)} L_{1}\left(k K_{a}+N+1\right) \\
& +a M_{2} M_{3} M_{4}\left\{\left\|x_{1}\right\|+M_{1}\|\phi(0)+F(0, \phi)\|\right. \\
& +M_{5} L_{1}\left(k K_{a}+N+1\right) \\
& +\frac{C_{1-\beta} \Gamma(1+\beta) a^{q \beta}}{\beta \Gamma(1+q \beta)} L_{1}\left(k K_{a}+N+1\right) \\
& \left.+M_{2} \int_{0}^{a}(a-\tau)^{q-1} g_{k K_{a}+N}(\tau) d \tau\right\}
\end{aligned}
$$




$$
\begin{aligned}
& +M_{2} \int_{0}^{a}(a-s)^{q-1} g_{k K_{a}+N}(s) d s \\
& =M_{5} L_{1} k K_{a}\left(1+a M_{2} M_{3} M_{4}\right) \\
& +M_{2}\left(1+a M_{2} M_{3} M_{4}\right) \int_{0}^{a}(a-s)^{q-1} g_{k K_{a}+N}(s) d s \\
& +\frac{C_{1-\beta} \Gamma(1+\beta) a^{q \beta}}{\beta \Gamma(1+q \beta)} L_{1}\left(k K_{a}+N+1\right)\left(1+a M_{2} M_{3} M_{4}\right) \\
& +M_{1}\|F(0, \phi)\|+M_{5} L_{1} N+M_{5} L_{1}+a M_{2} M_{3} M_{4}\left\|x_{1}\right\| \\
& +a M_{2} M_{3} M_{4}\|\phi(0)+F(0, \phi)\| \\
& +M_{5} L_{1} N a M_{2} M_{3} M_{4}+M_{5} L_{1} a M_{2} M_{3} M_{4} \\
& =M^{*}+\left(1+a M_{2} M_{3} M_{4}\right) \\
& \times\left[M_{5} L_{1} K_{a} k+M_{2}\left(\int_{0}^{a}(a-s)^{(q-1) /\left(q-q_{1}\right)} d s\right)^{1-q_{1}}\right. \\
& \times\left(\int_{0}^{a}\left(g_{k K_{a}+N}(s)\right)^{1 / q_{1}} d s\right)^{q_{1}} \\
& \left.+\frac{C_{1-\beta} \Gamma(1+\beta) a^{q \beta}}{\beta \Gamma(1+q \beta)} L_{1}\left(k K_{a}+N+1\right)\right] \\
& =M^{*}+\left(1+a M_{2} M_{3} M_{4}\right) \\
& \times\left[M_{5} L_{1} K_{a} k+M_{2}\left(\frac{1-q_{1}}{q-q_{1}} a^{\left(q-q_{1}\right) /\left(1-q_{1}\right)}\right)^{1-q_{1}}\right. \\
& \times\left\|g_{k K_{a}+N}\right\|_{L^{1 / q_{1}}[0, a]} \\
& \left.+\frac{C_{1-\beta} \Gamma(1+\beta) a^{q \beta}}{\beta \Gamma(1+q \beta)} L_{1}\left(k K_{a}+N+1\right)\right],
\end{aligned}
$$

where

$$
\begin{aligned}
M^{*}= & M_{1}\|F(0, \phi)\|+M_{5} L_{1} N+M_{5} L_{1}+a M_{2} M_{3} M_{4}\left\|x_{1}\right\| \\
& +a M_{2} M_{3} M_{4}\|\phi(0)+F(0, \phi)\| \\
& +M_{5} L_{1} N_{a} M_{2} M_{3} M_{4}+M_{5} L_{1} a M_{2} M_{3} M_{4} .
\end{aligned}
$$

Dividing on both sides by $k$ and taking the low limit, we get

$$
\begin{aligned}
& \left(1+a M_{2} M_{3} M_{4}\right) \\
& \times\left(L_{1} M_{0} K_{a}+M_{2}\left(\left(\frac{1-q_{1}}{q-q_{1}}\right) a^{\left(q-q_{1}\right) /\left(1-q_{1}\right)}\right)^{1-q_{1}} K_{a} \gamma\right. \\
& \left.+\frac{C_{1-\beta} \Gamma(1+\beta) a^{q \beta}}{\beta \Gamma(1+q \beta)} L_{1} K_{a}\right) \geq 1 .
\end{aligned}
$$

This contradicts (16). Hence for some positive number $k$, $\mathrm{Q} B_{k} \subseteq B_{k}$.
Now, we define operator $Q_{1}$ and $Q_{2}$ on $B_{k}$ as

$$
\begin{aligned}
\left(Q_{1} z\right)(t)= & \varphi(t) F(0, \phi)-F\left(t, \bar{z}_{t}+y_{t}\right) \\
& -\int_{0}^{t}(t-s)^{q-1} A S(t-s) F\left(s, \bar{z}_{s}+y_{s}\right) d s, \\
\left(Q_{2} z\right)(t)= & \int_{0}^{t}(t-s)^{q-1} S(t-s) \\
& \times\left[C u(s)+G\left(s, \bar{z}_{s}+y_{s}\right)\right] d s,
\end{aligned}
$$

for all $t \in[0, a]$, respectively.

We prove that $Q_{1}$ is contraction, while $Q_{2}$ is completely continuous.

Step 2. $Q_{1}$ is contraction.

Let $z_{1}, z_{2} \in B_{k}$. Then, for each $t \in[0, a]$, and by axiom (A)-(iii) and (15), we have

$$
\begin{aligned}
& \left\|Q_{1} z_{1}(t)-Q_{1} z_{2}(t)\right\| \\
& =\| \varphi(t) F(0, \phi)-F\left(t, \bar{z}_{1, t}+y_{t}\right) \\
& -\int_{0}^{t}(t-s)^{q-1} A S(t-s) F\left(s, \bar{z}_{1, s}+y_{s}\right) d s \\
& -\varphi(t) F(0, \phi)+F\left(t, \bar{z}_{2, t}+y_{t}\right) \\
& +\int_{0}^{t}(t-s)^{q-1} A S(t-s) F\left(s, \bar{z}_{2, s}+y_{s}\right) d s \| \\
& \leq\left\|F\left(t, \bar{z}_{1, t}+y_{t}\right)-F\left(t, \bar{z}_{2, t}+y_{t}\right)\right\| \\
& +\| \int_{0}^{t}(t-s)^{q-1} A S(t-s) \\
& \times\left(F\left(s, \bar{z}_{1, s}+y_{s}\right)-F\left(s, \bar{z}_{2, s}+y_{s}\right)\right) d s \| \\
& \leq\left\|A^{-\beta} A^{\beta} F\left(t, \bar{z}_{1, t}+y_{t}\right)-A^{-\beta} A^{\beta} F\left(t, \bar{z}_{2, t}+y_{t}\right)\right\| \\
& +\int_{0}^{t}(t-s)^{q-1} \\
& \times\left\|A^{1-\beta} S(t-s) A^{\beta}\left(F\left(s, \bar{z}_{1, s}+y_{s}\right)-F\left(s, \bar{z}_{2, s}+y_{s}\right)\right)\right\| d s \\
& \leq M_{5} L K_{a}\left\|\bar{z}_{1, t}-\bar{z}_{2, t}\right\|_{B} \\
& +\int_{0}^{a}(t-s)^{q-1} \frac{C_{1-\beta} q \Gamma(1+\beta)}{\Gamma(1+q \beta)}(t-s)^{-(1-\beta) q} \\
& \times L\left\|\bar{z}_{1, s}-\bar{z}_{2, s}\right\|_{B} d s \\
& \leq M_{5} L K_{a} \sup _{0 \leq s \leq a}\left\|z_{1}(s)-z_{2}(s)\right\| \\
& +\frac{C_{1-\beta} \Gamma(1+\beta) a^{q \beta}}{\beta \Gamma(1+q \beta)} L_{1} K_{a} \sup _{0 \leq s \leq a}\left\|z_{1}(s)-z_{2}(s)\right\|
\end{aligned}
$$




$$
\begin{aligned}
\leq & \left(M_{5} L K_{a}+\frac{C_{1-\beta} \Gamma(1+\beta) a^{q \beta}}{\beta \Gamma(1+q \beta)} L_{1} K_{a}\right) \\
& \times \sup _{0 \leq s \leq a}\left\|z_{1}(s)-z_{2}(s)\right\| .
\end{aligned}
$$

Thus

$$
\left\|Q_{1} z_{1}(t)-Q_{1} z_{2}(t)\right\|<\left\|z_{1}-z_{2}\right\|,
$$

and $Q_{1}$ is contraction.

Step 3. $Q_{2}$ is completely continuous.

Let $\left\{z_{n}\right\} \subseteq B_{k}$ with $z_{n} \rightarrow z$ in $B_{k}$, then for each $s \in$ $[0, a], \bar{z}_{n, s} \rightarrow \bar{z}_{s}$, and by $\left(H_{1}\right)$ and $\left(H_{2}\right)$-(i), we have

$$
\begin{gathered}
G\left(s, \bar{z}_{n, s}+y_{s}\right)-G\left(s, \bar{z}_{s}+y_{s}\right) \longrightarrow 0, \\
F\left(s, \bar{z}_{n, s}+y_{s}\right)-F\left(s, \bar{z}_{s}+y_{s}\right) \longrightarrow 0, \\
u_{n}(s)-u(s) \longrightarrow 0,
\end{gathered}
$$

as $n \rightarrow \infty$.

Since $\left\|G\left(s, \bar{z}_{n, s}+y_{s}\right)-G\left(s, \bar{z}_{s}+y_{s}\right)\right\| \leq 2 g_{k K_{a}+N(s)}$, then by the dominated convergence theorem we have

$$
\begin{aligned}
& \left\|Q_{2} z_{n}(t)-Q_{2} z(t)\right\| \\
& =\sup _{0 \leq t \leq a} \| \int_{0}^{t}(t-s)^{q-1} S(t-s) C u_{n}(s) d s \\
& \quad+\int_{0}^{t}(t-s)^{q-1} S(t-s) G\left(s, \bar{z}_{n, s}+y_{s}\right) d s \\
& \quad-\int_{0}^{t}(t-s)^{q-1} S(t-s) C u(s) d s \\
& \quad-\int_{0}^{t}(t-s)^{q-1} S(t-s) G\left(s, \bar{z}_{s}+y_{s}\right) d s \| \\
& \leq\left\|\int_{0}^{t}(t-s)^{q-1} S(t-s)\left(C u_{n}(s)-C u(s)\right) d s\right\| \\
& +\| \int_{0}^{t}(t-s)^{q-1} S(t-s) \\
& \quad \times\left(G\left(s, \bar{z}_{n, s}+y_{s}\right)-G\left(s, \bar{z}_{s}+y_{s}\right)\right) d s \| \longrightarrow 0,
\end{aligned}
$$

as $n \rightarrow \infty$, that is, $Q_{2}$ is continuous.
Next we prove that the family $\left\{Q_{2} z: z \in B_{k}\right\}$ is an equicontinuous family of functions. To do this, let $0 \leq t_{1}<$ $t_{2} \leq a$, then

$$
\begin{aligned}
& \left\|Q_{2} z\left(t_{2}\right)-Q_{2} z\left(t_{1}\right)\right\| \\
& =\| \int_{0}^{t_{2}}\left(t_{2}-s\right)^{q-1} S\left(t_{2}-s\right) C u(s) d s \\
& \quad+\int_{0}^{t_{2}}\left(t_{2}-s\right)^{q-1} S\left(t_{2}-s\right) G\left(s, \bar{z}_{s}+y_{s}\right) d s \\
& \quad-\int_{0}^{t_{1}}\left(t_{1}-s\right)^{q-1} S\left(t_{1}-s\right) C u(s) d s \\
& \quad-\int_{0}^{t_{1}}\left(t_{1}-s\right)^{q-1} S\left(t_{1}-s\right) G\left(s, \bar{z}_{s}+y_{s}\right) d s \| \\
& \leq M_{3} \int_{0}^{t_{1}}\left(t_{2}-s\right)^{q-1}\left\|\left(S\left(t_{2}-s\right)-S\left(t_{1}-s\right)\right) u(s)\right\| d s \\
& +M_{3} \int_{0}^{t_{1}}\left(\left(t_{2}-s\right)^{q-1}-\left(t_{1}-s\right)^{q-1}\right) \\
& \quad+\int_{0}^{t_{1}}\left(t_{2}-s\right)^{q-1} \\
& \quad \times\left\|\left(S\left(t_{2}-s\right)-S\left(t_{1}-s\right)\right) G\left(s, \bar{z}_{s}+y_{s}\right)\right\| d s \\
& +\int_{0}^{t_{1}}\left(\left(t_{2}-s\right)^{q-1}-\left(t_{1}-s\right)^{q-1}\right) \\
& +\int_{t_{1}}^{t_{2}}\left(t_{2}-s\right)^{q-1}\left\|S\left(t_{2}-s\right)\right\|\left\|G\left(s, \bar{z}_{s}+y_{s}\right)\right\| d s . \\
& \quad \times\left\|S\left(t_{1}-s\right)\right\|\left\|G\left(s, \bar{z}_{s}+y_{s}\right)\right\| d s \\
& M_{3} \int_{t_{1}}^{t_{2}}\left(t_{2}-s\right)^{q-1}\left\|S\left(t_{2}-s\right)\right\|\|u(s)\| d s
\end{aligned}
$$

Noting that

$$
\begin{aligned}
\|u(s)\| \leq M_{4}[ & \left\|x_{1}\right\|+M_{1}\|\phi(0)+F(0, \phi)\|+\left\|F\left(a, x_{a}\right)\right\| \\
& +\int_{0}^{a}(a-s)^{q-1}\left\|S(a-s) F\left(s, x_{s}\right)\right\| d s \\
& \left.+\int_{0}^{a}(a-s)^{q-1}\left\|S(a-s) G\left(s, x_{s}\right)\right\| d s\right] \\
\leq M_{4}\left[\left\|x_{1}\right\|+M_{1}\|\phi(0)+F(0, \phi)\|\right. & \\
+ & \left\|F\left(a, x_{a}\right)\right\|+M_{5} L_{1}\left(k K_{a}+N+1\right) \\
+ & \frac{C_{1-\beta} \Gamma(1+\beta) a^{q \beta}}{\beta \Gamma(1+q \beta)} L_{1}\left(k K_{a}+N+1\right)
\end{aligned}
$$




$$
\begin{gathered}
\quad+M_{2}\left(\frac{1-q_{1}}{q-q_{1}} a^{\left(q-q_{1}\right) /\left(1-q_{1}\right)}\right)^{1-q_{1}} \\
\left.\times\left\|g_{k K_{a}+N}\right\|_{L^{1 / q_{1}}[0, a]}\right] \\
\int_{0}^{t}\left\|(t-s)^{q-1} G\left(s, \bar{z}_{k, s}+y_{s}\right)\right\| d s \\
\leq \int_{0}^{t}(t-s)^{q-1} g_{k K_{a}+N}(s) d s \\
\leq \int_{0}^{a}(a-s)^{q-1} g_{k K_{a}+N}(s) d s \\
\leq\left(\left(\frac{1-q_{1}}{q-q_{1}}\right) a^{\left(q-q_{1}\right) /\left(1-q_{1}\right)}\right)^{1-q_{1}}\left\|g_{k K_{a}+N}\right\|_{L^{1 / q_{1}[0, a]}} .
\end{gathered}
$$

We see that $\left\|Q_{2} z\left(t_{2}\right)-Q_{2} z\left(t_{1}\right)\right\|$ tends to zero independently of $z \in B_{k}$ as $t_{2} \rightarrow t_{1}$ since for $t \in[0, a]$ and any bounded subsets $D \subset X, t \rightarrow\{S(t) x: x \in D\}$ is equicontinuous.

Hence, $Q_{2}$ maps $B_{k}$ into an equicontinuous family functions.

It remains to prove that $V(t)=\left\{\left(Q_{2} z\right)(t): z \in B_{k}\right\}$ is relatively compact in $X$. let $0 \leq t \leq a$ be fixed, $0<\epsilon<t$, for $z \in B_{k}$, we define $\Pi=Q_{2} B_{k}$ and $\Pi(t)=\left\{Q_{2} z(t) \mid z \in B_{k}\right\}$, for $t \in[0, a]$.

Clearly, $\Pi(0)=\left\{Q_{2} z(0) \mid z \in B_{k}\right\}=\{0\}$ is compact, and hence, it is only to consider $0<t \leq a$. For each $h \in(0, t), t \in$ $(0, a]$, arbitrary $\delta>0$, define

$$
\Pi_{h, \delta}(t)=\left\{Q_{2, h, \delta} z(t) \mid z \in B_{k}\right\},
$$

where

$$
\begin{aligned}
Q_{2, h, \delta} z(t)=\int_{0}^{t-h} \int_{\delta}^{\infty}(t-s)^{q-1} \theta \xi_{q}(\theta) q T\left((t-s)^{q} \theta\right) \\
\times\left[C u(s)+G\left(s, \bar{z}_{s}+y_{s}\right)\right] d \theta d s .
\end{aligned}
$$

Then the sets $\left\{Q_{2, h, \delta} z(t) \mid z \in B_{k}\right\}$ are relatively compact in $X$ since the condition $\left(H_{4}\right)$. It comes from the following inequalities:

$$
\begin{aligned}
& \left\|Q_{2} z(t)-Q_{2, h, \delta} z(t)\right\| \\
& =\| \int_{0}^{t} \int_{0}^{\infty}(t-s)^{q-1} \theta \xi_{q}(\theta) q T\left((t-s)^{q} \theta\right) \\
& \quad \times\left[C u(s)+G\left(s, \bar{z}_{s}+y_{s}\right)\right] d \theta d s \\
& -\int_{0}^{t-h} \int_{\delta}^{\infty}(t-s)^{q-1} \theta \xi_{q}(\theta) q T\left((t-s)^{q} \theta\right) \\
& \quad \times\left[C u(s)+G\left(s, \bar{z}_{s}+y_{s}\right)\right] d \theta d s \|
\end{aligned}
$$

$$
\begin{aligned}
& =\| \int_{0}^{t} \int_{0}^{\delta}(t-s)^{q-1} \theta \xi_{q}(\theta) q T\left((t-s)^{q} \theta\right) \\
& \times\left[C u(s)+G\left(s, \bar{z}_{s}+y_{s}\right)\right] d \theta d s \\
& +\int_{0}^{t} \int_{\delta}^{\infty}(t-s)^{q-1} \theta \xi_{q}(\theta) q T\left((t-s)^{q} \theta\right) \\
& \times\left[C u(s)+G\left(s, \bar{z}_{s}+y_{s}\right)\right] d \theta d s \\
& -\int_{0}^{t-h} \int_{\delta}^{\infty}(t-s)^{q-1} \theta \xi_{q}(\theta) q T\left((t-s)^{q} \theta\right) \\
& -\int_{0}^{t-h} \int_{\delta}^{\infty}(t-s)^{q-1} \theta \xi_{q}(\theta) q T\left((t-s)^{q} \theta\right) \\
& \times\left[C u(s)+G\left(s, \bar{z}_{s}+y_{s}\right)\right] d \theta d s \| \\
& \leq M M_{3}\|u(s)\| q \int_{0}^{t}(t-s)^{q-1} d s \int_{0}^{\delta} \theta \xi_{q}(\theta) d \theta \\
& +M q \int_{0}^{t}(t-s)^{q-1} g_{k K_{a}+N}(s) d s \int_{0}^{\delta} \theta \xi_{q}(\theta) d \theta \\
& +M M_{3}\|u(s)\| \int_{t-h}^{t}(t-s)^{q-1} d s \cdot q \int_{\delta}^{\infty} \theta \xi_{q}(\theta) d \theta \\
& +M \int_{t-h}^{t}(t-s)^{q-1} g_{k K_{a}+N}(s) d s \cdot q \int_{\delta}^{\infty} \theta \xi_{q}(\theta) d \theta \\
& \leq\left\{M M _ { 3 } M _ { 4 } \left[\left\|x_{1}\right\|+M_{1}\|\phi(0)+F(0, \phi)\|\right.\right. \\
& +\left\|F\left(a, x_{a}\right)\right\|+M_{5} L_{1}\left(k K_{a}+N+1\right) \\
& +\frac{C_{1-\beta} \Gamma(1+\beta) a^{q \beta}}{\beta \Gamma(1+q \beta)} L_{1}\left(k K_{a}+N+1\right) \\
& +M\left(\left(\frac{1-q_{1}}{q-q_{1}}\right) a^{\left(q-q_{1}\right) /\left(1-q_{1}\right)}\right)^{1-q_{1}} \\
& \left.\times\left\|g_{k K_{a}+N}\right\|_{L^{1 / q_{1}}[0, a]}\right] a^{q} \\
& \left.+M\left(\left(\frac{1-q_{1}}{q-q_{1}}\right) a^{\left(q-q_{1}\right) /\left(1-q_{1}\right)}\right)^{1-q_{1}}\left\|g_{k K_{a}+N}\right\|_{L^{1 / q_{1}}[0, a]}\right\} q \\
& \times \int_{0}^{\delta} \theta \xi_{q}(\theta) d \theta+M\left(\left(\frac{1-q_{1}}{q-q_{1}}\right) h^{\left(q-q_{1}\right) /\left(1-q_{1}\right)}\right)^{1-q_{1}} \\
& \times\left\|g_{k K_{a}+N}\right\|_{L^{1 / q_{1}[0, a]}} q \int_{0}^{\infty} \theta \xi_{q}(\theta) d \theta
\end{aligned}
$$

Therefore, $\Pi(t)=\left\{Q_{2} z(t) \mid z \in B_{k}\right\}$ is relatively compact in $X$ for all $t \in[0, a]$.

Thus, the continuity of $Q_{2}$ and relatively compact of $\left\{Q_{2} z(t) \mid z \in B_{k}\right\}$ imply that $Q_{2}$ is a completely continuous operator. 
These arguments enable us to conclude that $Q=Q_{1}+Q_{2}$ is a condense mapping on $B_{k}$, and by the fixed point theorem of Sadovskii there exists a fixed point $z(\cdot)$ for $Q$ on $B_{k}$. In fact, by Step 1-Step 3 and Lemma 3, we can conclude that $Q=Q_{1}+$ $Q_{2}$ is continuous and takes bounded sets into bounded sets. Meanwhile, it is easy to see $\alpha\left(Q_{2}\left(B_{k}\right)\right)=0$ since $Q_{2}\left(B_{k}\right)$ is relatively compact. Since $\left.Q_{1}\left(B_{k}\right)\right) \subseteq B_{k}$ and $\alpha\left(Q_{2}\left(B_{k}\right)\right)=0$, we can obtain $\alpha\left(Q\left(B_{k}\right)\right) \leq \alpha\left(Q_{1}\left(B_{k}\right)\right)+\alpha\left(Q_{2}\left(B_{k}\right)\right) \leq \alpha\left(B_{k}\right)$ for every bounded set $B_{k}$ of $X$ with $\alpha\left(B_{k}\right)>0$, that is, $Q=$ $Q_{1}+Q_{2}$ is a condense mapping on $B_{k}$. If we define $x(t)=$ $z(t)+y(t),-\infty<t \leq a$, it is easy to see that $x(\cdot)$ is a mild solution of (1) satisfying $x_{0}=\phi, x(a)=x_{1}$. Then the proof is completed.

Remark 8. In order to describe various real-world problems in physical and engineering sciences subject to abrupt changes at certain instants during the evolution process, impulsive fractional differential equations always have been used in the system model. So we can also consider the complete controllability for (1) with impulses.

Remark 9. Since the complete controllability steers the systems to arbitrary final state while approximate controllability steers the system to arbitrary small neighborhood of final state. In view of the definition of approximate controllability in [28], we can deduce that the considered systems (1) is also approximate controllable on the interval $[0, a]$.

\section{An Example}

As an application of Theorem 7, we consider the following system:

$$
\begin{gathered}
\frac{\partial^{2 / 3}}{\partial t^{2 / 3}}\left[z(t, x)+\int_{-\infty}^{t} \int_{0}^{\pi} b(s-t, y, x) z(s, y) d y d s\right] \\
\quad-\frac{\partial^{2}}{\partial x^{2}} z(t, x) \\
=C u(t)+a_{0}(x) z(t, x) \\
\quad+\int_{-\infty}^{t} a_{1}(s, t) z(s, x) d s+a_{2}(t, x), \\
\quad 0 \leq t \leq a, \quad 0 \leq x \leq \pi, \quad \\
z(t, 0)=z(t, \pi)=0, \quad z(\vartheta, x)=\phi(\vartheta, x), \quad \vartheta \leq 0 .
\end{gathered}
$$

To write system (40) to the form of (1), let $X=L^{2}([0, \pi])$ and $A$ defined by $A f=-f^{\prime \prime}$ with domain $D(A)=\{f(\cdot) \epsilon$ $X: f, f^{\prime}$ absolutely continuous, $\left.f^{\prime \prime} \in X, f(0)=f(\pi)=0\right\}$, the $-A$ generates a uniformly bounded analytic semigroup which satisfies the condition $\left(H_{0}\right)$. Furthermore, $A$ has a discrete spectrum, the eigenvalues are $-n^{2}, n \in N$, with the corresponding normalized eigenvectors $z_{n}(x)=$ $(2 / \pi)^{1 / 2} \sin (n x)$. Then the following properties hold.

(i) If $A \in D(A)$, then

$$
A f=\sum_{n=1}^{\infty} n^{2}\left\langle f, z_{n}\right\rangle z_{n}
$$

(ii) For each $f \in X$,

$$
A^{-1 / 2} f=\sum_{n=1}^{\infty} \frac{1}{n}\left\langle f, z_{n}\right\rangle z_{n}
$$

In particular, $\left\|A^{-1 / 2}\right\|=1$.

(iii) The operator $A^{1 / 2}$ is given by

$$
A^{1 / 2} f=\sum_{n=1}^{\infty} n\left\langle f, z_{n}\right\rangle z_{n}
$$

on the space $D\left(A^{1 / 2}\right)=\left\{f(\cdot) \in X, A^{1 / 2} f \in X\right\}$.

Here we take the phase space $B=C_{0} \times L^{2}(g, X)$, which contains all classes of functions $\phi:(-\infty, 0] \rightarrow X$ such that $\phi$ is Lebesgue measurable and $g(\cdot)\|\phi(\cdot)\|^{2}$ is Lebesgue integrable on $(-\infty, 0)$ where $g:(-\infty, 0) \rightarrow R$ is a positive integrable function. The seminorm in $B$ is defined by

$$
\|\phi\|_{B}=\|\phi(0)\|+\left(\int_{-\infty}^{0} g(\vartheta)\|\phi(\vartheta)\|^{2} d \vartheta\right)^{1 / 2} .
$$

From [41], under some conditions $B$ is a phase space verifying (A), (B), (C), and in this case $K(t)=1+\left(\int_{-t}^{0} g(\vartheta) d \vartheta\right)^{1 / 2}$ (see [41] for the details).

We assume the following conditions hold.

(a) The function $b$ is measurable and $\int_{0}^{\pi} \int_{-\infty}^{0} \int_{0}^{\pi}\left(b^{2}(\vartheta, y\right.$, $x) / g(\vartheta)) d y d \vartheta d x<\infty$.

(b) The function $(\partial / \partial x) b(\vartheta, y, x)$ is measurable, $b(\vartheta, y$, $0)=b(\vartheta, y, \pi)=0$ and let $N_{1}=\int_{0}^{\pi} \int_{-\infty}^{0} \int_{0}^{\pi}(1 /$ $g(\vartheta))((\partial / \partial x) b(\vartheta, y, x))^{2} d y d \vartheta d x<\infty$.

(c) The function $a_{0}(\cdot) \in L^{\infty}([0, \pi]), a(\cdot)$ is measurable, with $\int_{-\infty}^{0}\left(a_{1}^{2}(\vartheta)\right) / g(\vartheta) d \vartheta<\infty$, the function $a_{2}(t, \cdot) \in$ $L^{2}([0, \pi])$ for each $t \geq 0$ is measurable in $t$.

(d) The function $\phi$ defined by $\phi(\vartheta)(x)=\phi(\vartheta, x)$ belongs to $B$.

(e) The linear operator $W: U \rightarrow X$ is defined by

$$
W u=\int_{0}^{a}(a-s)^{-1 / 3} S(a-s) C u(s) d s
$$

and has a bounded invertible operator $W^{-1}$ defined $L^{2}([0$, a]); $U) / \operatorname{ker} W$.

We define $F, G:[0, a] \times B \rightarrow X$ by $F(t, \phi)=Z_{1}(\phi)$ and $G(t, \phi)=Z_{2}(\phi)+h(t)$, where

$$
\begin{gathered}
Z_{1}(\phi)=\int_{-\infty}^{0} \int_{0}^{\pi} b(\vartheta, y, x) \phi(\vartheta, x) d y d \vartheta, \\
Z_{2}(\phi)=a_{0}(x) \phi(0, x)+\int_{-\infty}^{0} a_{1}(\vartheta) \phi(\vartheta, x) d \vartheta, \\
h(t)=a_{2}(t, \cdot) .
\end{gathered}
$$


From (a) and (c) it is clear that $Z_{1}$ and $Z_{2}$ are bounded linear operators on $B$. Furthermore, $Z_{1}(\phi) \in D\left(A^{1 / 2}\right)$, and $\left\|A^{1 / 2} Z_{1}\right\| \leq N_{1}$. In fact, from the definition of $Z_{1}$ and (b) it follows that $\left\langle Z_{1}(\phi), z_{n}\right\rangle=(1 / n)(2 / \pi)^{1 / 2}\langle Z(\phi), \operatorname{con}(n x)\rangle$, where $Z(\phi)=\int_{-\infty}^{0} \int_{0}^{\pi}(\partial / \partial x) b(\vartheta, y, x) \phi(\vartheta, x) d \vartheta$. From (b) we know that $Z: B \rightarrow X$ is a bounded linear operator with $\|Z\| \leq N_{1}$. Hence $\left\|A^{1 / 2} Z_{1}(\phi)\right\|=\|Z(\phi)\|$, which implies the assertion. Therefore, from Theorem 7 , the system (40) is completely controllable on $[0, a]$ under the above assumptions.

\section{Conclusion}

In this paper, by using the uniformly boundedness, analyticity, and equicontinuity of characteristic solution operators and the Sadovskii fixed point theorem, we obtained the complete controllability of the abstract neutral fractional differential systems with unbounded delay in a Banach space. It shows that the compactness of the characteristic solution operators can be weakened to equicontinuity. Our theorem guarantees the effectiveness of complete controllability results under some weakly compactness conditions.

\section{Acknowledgments}

The authors are highly grateful for the referee's careful reading and comments on this paper. The present Project is supported by NNSF of China Grant no. 11271087, no. 61263006, and Guangxi Scientific Experimental (China-ASEAN Research) Centre no. 20120116.

\section{References}

[1] K. Diethelm and A. D. Freed, "On the solution of nonlinear fractional order differential equations used in the modeling of viscoelasticity," in Scientific Computing in Chemical Engineering II-Computational Fluid Dynamics, Reaction Engineering and Molecular Properties, F. Keil, W. Machens, H. Voss, and J. Werther, Eds., pp. 217-224, Springer, Heidelberg, Germany, 1999.

[2] N. U. Ahmed, Dynamic Systems and Control with Application, World Scientific, Hackensack, NJ, USA, 2006.

[3] Y. A. Rossikhin and M. V. Shitikova, "Applications of fractional calculus to dynamic problems of linear and nonlinear hereditary mechanics of solids," Applied Mechanics Reviews, vol. 50, no. 1, pp. 15-67, 1997.

[4] L. Debnath, "Recent applications of fractional calculus to science and engineering," International Journal of Mathematics and Mathematical Sciences, vol. 2003, no. 54, pp. 3413-3442, 2003.

[5] R. Hilfer, Applications of Fractional Calculus in Physics, World Scientific, Singapore, 2000.

[6] A. A. Kilbas, H. M. Srivastava, and J. J. Trujillo, Theory and Applications of Fractional Differential Equations, vol. 204 of North-Holland Mathematics Studies, Elsevier Science, Amsterdam, The Netherland, 2006.

[7] K. S. Miller and B. Ross, An Introduction to Fractional Calculus and Fractional Differential Equation, Wiley, New York, NY, USA, 1993.

[8] I. Podlubny, Fractional Differential Equations, vol. 198, Academic Press, San Diego, Calif, USA, 1999.
[9] V. Lakshmikantham, S. Leela, and J. D. Vasundhara, Theory of Fractional Dynamics Systems, Cambridge Scientific, Cambridge, UK, 2009.

[10] V. E. Tarasov, Fractional Dynamics: Applications of Fractional Calculus to Dynamics of Particles, Fields and Media, Springer, Heidelberg, Germany, 2010.

[11] F. Wang, "Existence and uniqueness of solutions for a nonlinear fractional differential equation," Journal of Applied Mathematics and Computing, vol. 39, no. 1-2, pp. 53-67, 2012.

[12] F. Wang, Z. H. Liu, and P. Wang, "Analysis of a system for linear fractional equaitons," Journal of Applied Mathematics, vol. 2012, Article ID 193061, 21 pages, 2012.

[13] F. Wang and Z. H. Liu, "Anti-periodic fractional boundary value problems for nonlinear differential equations of fractional order," Advances in Difference Equation, vol. 2012, artilce 116, 12 pages, 2012.

[14] R. P. Agarwal, M. Belmekki, and M. Benchohra, "A survey on semilinear differential equations and inclusions involving Riemann-Liouville fractional derivative," Advances in Difference Equations, vol. 2009, Article ID 981728, 47 pages, 2009.

[15] M. M. El-Borai, "Some probability densities and fundamental solutions of fractional evolution equations," Chaos, Solitons and Fractals, vol. 14, no. 3, pp. 433-440, 2002.

[16] Y. Zhou and F. Jiao, "Nonlocal Cauchy problem for fractional evolution equations," Nonlinear Analysis: Real World Applications, vol. 11, no. 5, pp. 4465-4475, 2010.

[17] K. Balachandran and R. Sakthivel, "Controllability of functional semilinear integrodifferential systems in Banach spaces," Journal of Mathematical Analysis and Applications, vol. 255, no. 2, pp. 447-457, 2001.

[18] K. Balachanadran and E. R. Anandhi, "Controllability of neurtal functional integrodifferential infinite delay systems in Banach spaces," Nonlinear Analysis, vol. 61, pp. 405-423, 2005.

[19] J. R. Wang and Y. Zhou, "Complete controllability of fractional evolution systems," Communications in Nonlinear Science and Numerical Simulation, vol. 17, no. 11, pp. 4346-4355, 2012.

[20] J. R. Wang, Z. Fan, and Y. Zhou, "Nonlocal controllability of semilinear dynamic systems with fractional derivative in Banach spaces," Journal of Optimization Theory and Applications, vol. 154, no. 1, pp. 292-302, 2012.

[21] J. Wang and Y. Zhou, "A class of fractional evolution equations and optimal controls," Nonlinear Analysis: Real World Applications, vol. 12, no. 1, pp. 262-272, 2011.

[22] J. Wang, Y. Zhou, and W. Wei, "A class of fractional delay nonlinear integrodifferential controlled systems in Banach spaces," Communications in Nonlinear Science and Numerical Simulation, vol. 16, no. 10, pp. 4049-4059, 2011.

[23] J. Wang and Y. Zhou, "Analysis of nonlinear fractional control systems in Banach spaces," Nonlinear Analysis: Theory, Methods and Applications A, vol. 74, no. 17, pp. 5929-5942, 2011.

[24] R. Sakthivel, N. I. Mahmudov, and Juan. J. Nieto, "Controllability for a class of fractional-order neutral evolution control systems," Applied Mathematics and Computation, vol. 218, no. 20, pp. 10334-10340, 2012.

[25] Y. Ren, L. Hu, and R. Sakthivel, "Controllability of impulsive neutral stochastic functional differential inclusions with infinite delay," Journal of Computational and Applied Mathematics, vol. 235, no. 8, pp. 2603-2614, 2011.

[26] R. Sakthivel, Y. Ren, and N. I. Mahmudov, "On the approximate controllability of semilinear fractional differential systems," Computers and Mathematics with Applications, vol. 62, no. 3, pp. 1451-1459, 2011. 
[27] K. Rykaczewski, "Approximate controllability of differential inclusions in Hilbert spaces," Nonlinear Analysis: Theory, Methods and Applications A, vol. 75, no. 5, pp. 2701-2712, 2012.

[28] R. Sakthivel, S. Suganya, and S. M. Anthoni, "Approximate controllability of fractional stochastic evolution equations," Computers and Mathematics with Applications, vol. 63, no. 3, pp. 660-668, 2012.

[29] R. Sakthivel and Y. Ren, "Complete controllability of stochastic evolution equations with jumps," Reports on Mathematical Physics, vol. 68, no. 2, pp. 163-174, 2011.

[30] Y.-K. Chang and D. N. Chalishajar, "Controllability of mixed Volterra-Fredholm-type integro-differential inclusions in Banach spaces," Journal of the Franklin Institute, vol. 345, no. 5, pp. 499-507, 2008.

[31] X. Fu, "Controllability of neutral functional differential systems in abstract space," Applied Mathematics and Computation, vol. 141, no. 2-3, pp. 281-296, 2003.

[32] S. Ji, G. Li, and M. Wang, "Controllability of impulsive differential systems with nonlocal conditions," Applied Mathematics and Computation, vol. 217, no. 16, pp. 6981-6989, 2011.

[33] Z. Tai, "Controllability of fractional impulsive neutral integrodifferential systems with a nonlocal Cauchy condition in Banach spaces," Applied Mathematics Letters, vol. 24, no. 12, pp. 21582161, 2011.

[34] E. Hernández M. and D. O’Regan, "Controllability of VolterraFredholm type systems in Banach spaces," Journal of the Franklin Institute, vol. 346, no. 2, pp. 95-101, 2009.

[35] E. Hernández, D. O’Regan, and K. Balachandran, “On recent developments in the theory of abstract differential equations with fractional derivatives," Nonlinear Analysis: Theory, Methods and Applications A, vol. 73, no. 10, pp. 3462-3471, 2010.

[36] Y. Zhou and F. Jiao, "Existence of mild solutions for fractional neutral evolution equations," Computers and Mathematics with Applications, vol. 59, no. 3, pp. 1063-1077, 2010.

[37] M. A. Krasnoselskii, Topological Methods in the Theory of Nonlinear Integral Equations, Pergano Press, New York, NY, USA, 1964.

[38] B. N. Sadovskiı̌, “On a fixed point principle," Akademija Nauk SSSR, vol. 1, no. 2, pp. 74-76, 1967.

[39] P. Zhao and J. Zheng, "Remarks on Hausdorff measure and stability for the $p$-obstacle problem," Proceedings Mathematical Sciences, vol. 122, no. 1, pp. 129-137, 2012.

[40] J. K. Hale and J. Kato, "Phase space for retarded equations with infinite delay," Funkcialaj Ekvacioj, vol. 21, no. 1, pp. 11-41, 1978.

[41] Y. Hino, S. Murakami, and T. Naito, Functional-differential equations with infinite delay, vol. 1473 of Lecture Notes in Mathematics, Springer-Verlag, Berlin, 1991.

[42] C.-M. Marle, Mesures et Probabilités, Hermann, Paris, France, 1974. 


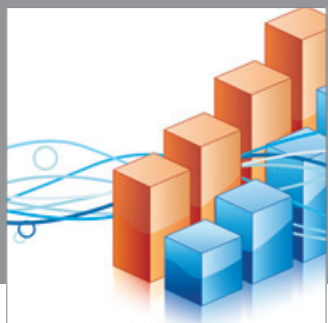

Advances in

Operations Research

mansans

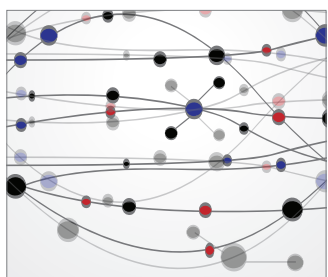

The Scientific World Journal
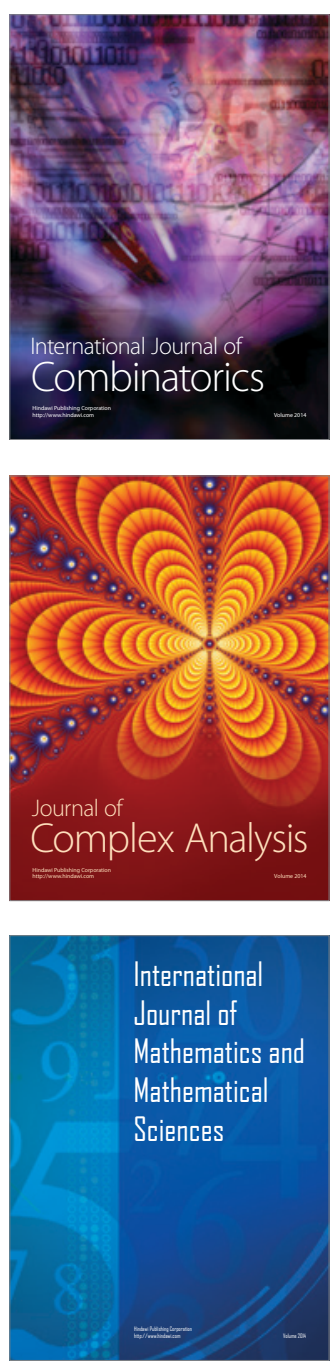
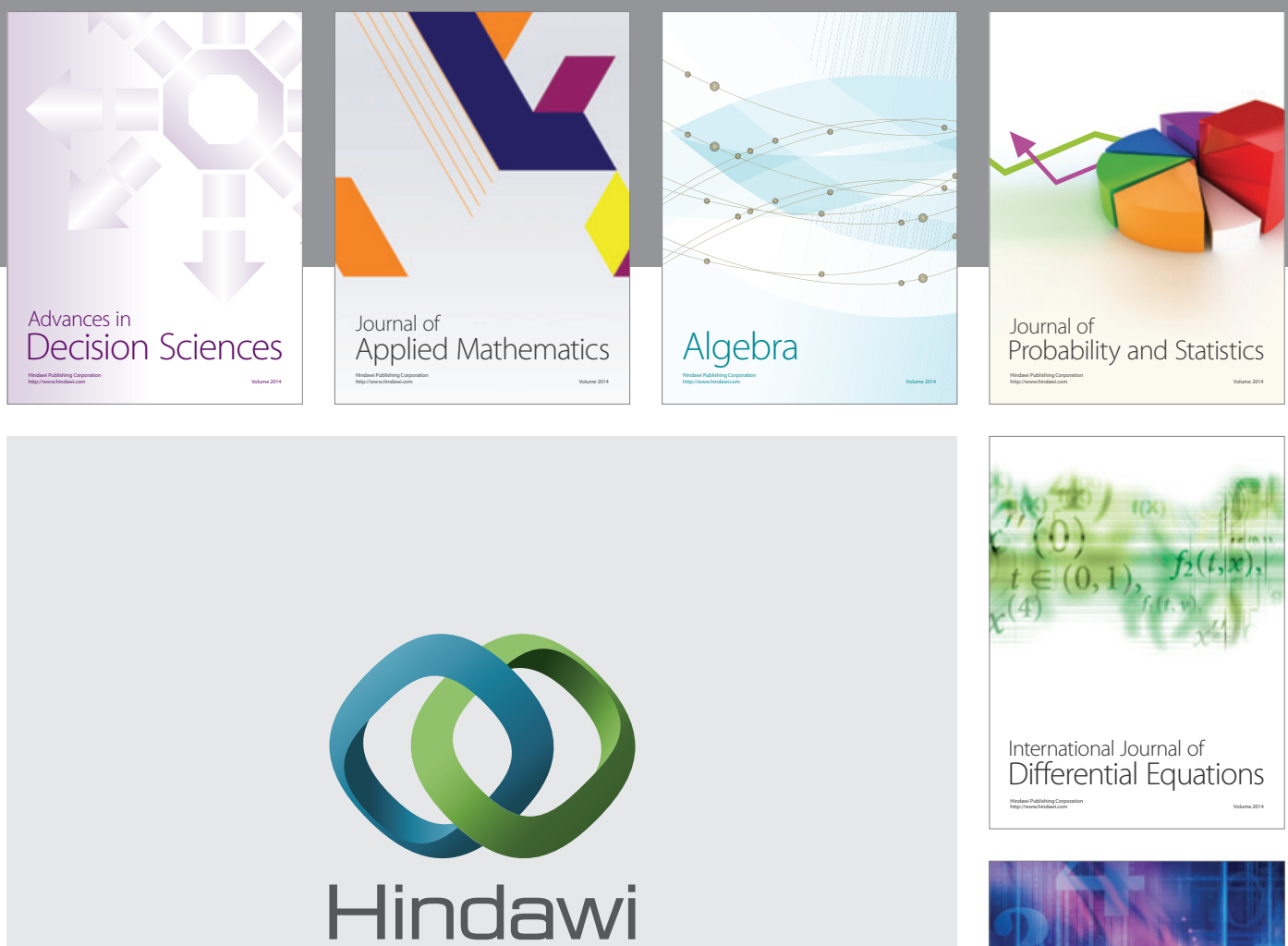

Submit your manuscripts at http://www.hindawi.com
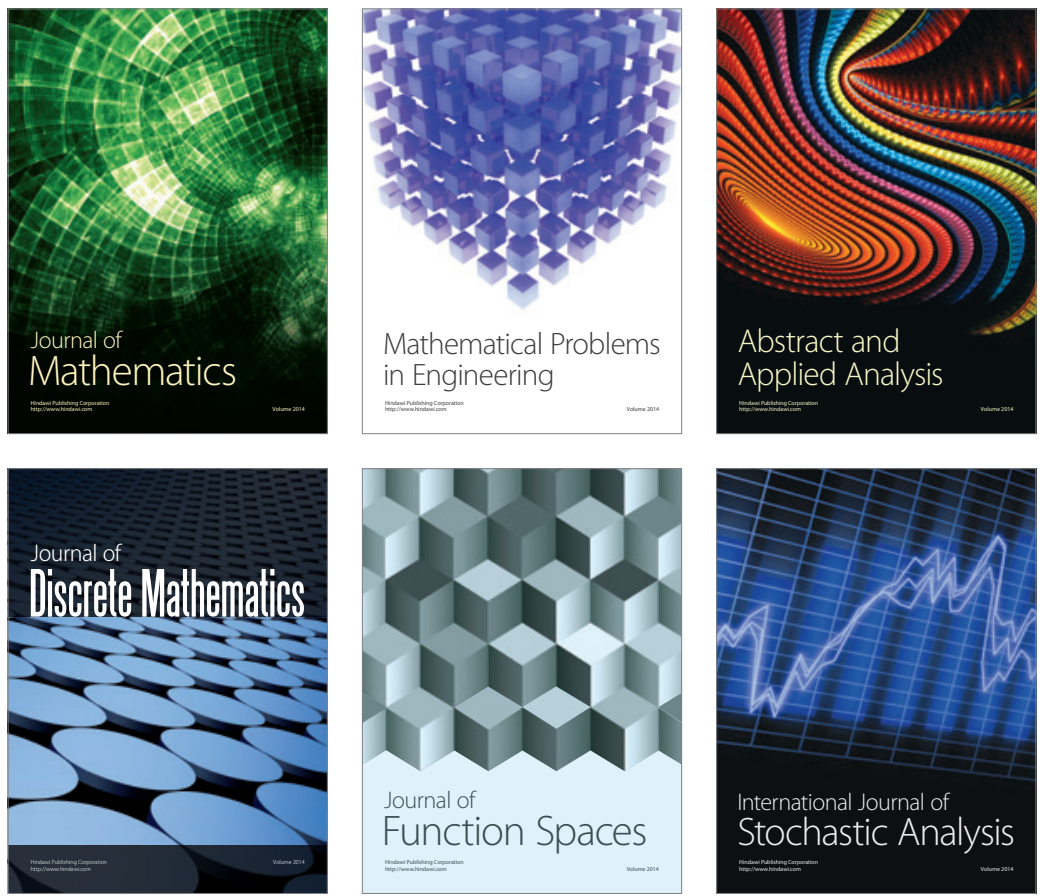

Journal of

Function Spaces



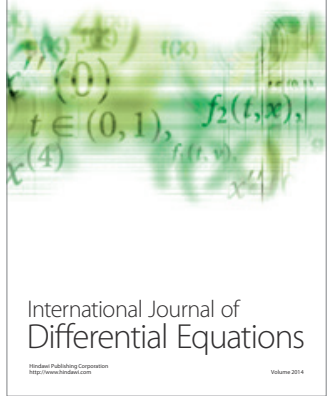
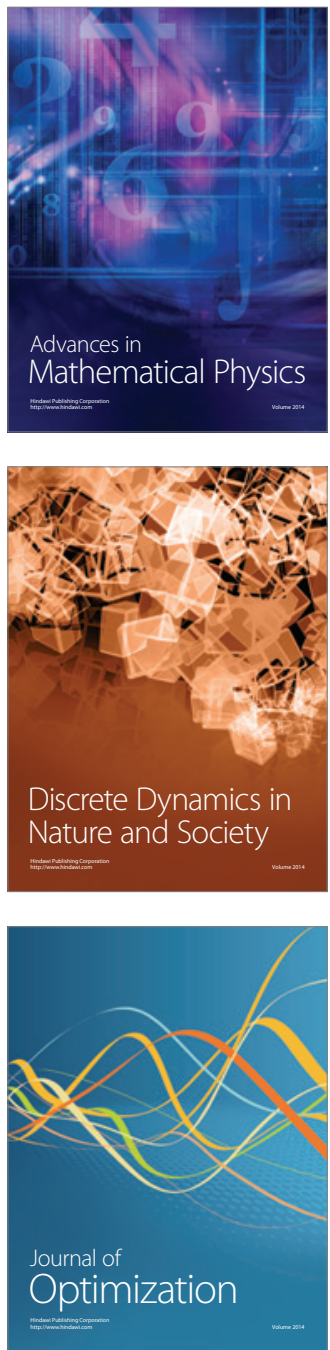